\title{
The Effect of Acceptance and Commitment Therapy in Reducing the Anxiety of Female Teenagers of Tehran City
}

\author{
Melody Shahab ${ }^{1}$
}

\begin{abstract}
:
The present study aimed to determine the effectiveness of the "Acceptance and Commitment Therapy (ACT)" in reducing symptoms of anxiety in adolescent girls in Tehran. 30 girls of 14 and 18 years of age, who were referrers to the Ertebat ,Hamdardi ,Movaffaghiat Psychology Center of Tehran because of symptoms of anxiety, were selected and randomly assigned to a test group and a control group each one including 15 patients. The Acceptance and Commitment Therapy Protocol of 12 sessions were conducted on the experimental group. The control group received no intervention. The research method used in the study was the pretest- posttest semiexperimental method with the control group. To measure the symptoms of anxiety in the study, the dass-42 questionnaires were used and to analyze the data the SPANQVA test was applied. The data analysis results indicated the effectiveness of the Acceptance and Commitment Therapy in reducing the adolescent girls' symptoms of anxiety. The results indicate that the improvement of psychological flexibility which is the major component of the Acceptance and Commitment Therapy has a considerable effect on the dependent variable.
\end{abstract}

Keywords: Acceptance and Commitment Therapy (ACT)", psychological flexibility, anxiety

General anxiety disorder $(\mathrm{GDA})_{3}$, is one of the most frequent anxiety disorders having a high simultaneousness with the other anxiety disorders. The characteristic feature of that disorder is an extremist and uncontrollable anxiety, associated with some anxiety symptoms.

For curing that disorder, beyond pharmaceutical therapy, numerous psychological therapies have also been developed through successive years. The first generation of the behavioral approaches in contrast to the earlier psycho-analytical approaches was suggested on the foundation of classic conditional views, in decades of 1950 , and 1960 . The $2^{\text {nd }}$ generation of those therapies was come to existence under the title of behavioral-cognitive therapy, with a greater emphasis on the cognitive aspects, up to the decade of 1990. Most precision studies related to the anxiety disorders have been conducted using cognitive-behavioral techniques, seemingly are influential both in short term, and in long term too (Sadook \& Sadook, translated by Mr. FarzinRezaiee,2008).

${ }^{1}$ Islamic Azad University at Kish, International uni.

(C) 2015 I M Shahab; licensee IJIP. This is an Open Access Research distributed under the terms of the Creative Commons Attribution License (http://creativecommons.org/licenses/by/2.0), which permits unrestricted use, distribution, and reproduction in any Medium, provided the original work is properly cited. 


\section{The Effect of Acceptance and Commitment Therapy in Reducing the Anxiety of Female Teenagers of Tehran City}

But today, we are confronted with the $3^{\text {rd }}$ generation of those therapies, which they can be called and classified under the general title of the models based on acceptance; Such as acceptance \& commitment therapy $(\mathrm{ACT})^{14}$, these therapies are trying to increase psychological relation/link of the individual with thoughts \& sensibilities/sympathies (Izadi \& Abedi, 2014).

In ACT the essential goal is creating psychological flexibility; i.e. producing practical ability to choose among different options whichever is more appropriate, rather than an action merely for avoiding the thoughts, sympathies \& sensibilities, impressions, and or disturbing inclinations/tendencies, which is in fact, imposed with biasing on the individual. In recent years, the efficiency of acceptance \& commitment therapy in curing general anxiety disorder has been confirmed (Ramer \& Orsilo $\left.{ }^{5}, 2007\right)$. It is expected that, developing and fostering «acceptance» in the referee subjects, would entail in decrease of «cognitive avoidance», and development and growth of the «value-based life» would also cause increase in the individual's practice and action in different fields of his life (Eifert\& Forsyth ${ }^{6}, 2005$ ). Or self-personal episode. In the $5^{\text {th }}$ step assistance and help is given to the individual to recognize his personal essential values, and finally producing impetus \& motive to the performing as obligatory that is, doing activity towards the specified and defined objectives and values, along with accepting the mental experiences (Sawdara, 2007).

The main goal of the acceptance \& commitment therapy is mostly shifting orientation of focus and efforts of the referee subjects far away from idle objectives \& goals ( like as decreasing unpleasant thoughts and sensibilities \& sympathies) towards actions based on their demands of a desirable life. The entire efforts of a consultant for the growth \& fostering a «value based life» is to help referee (subjects) to find their own favorable living method \& procedure, and proceed merely with that method. All of the therapeutic efforts shall focus on behaviors ahead of values chosen by reference/referral authorities (Eifert \& Forysth, 2005). Accordingly the intended question here in the present research is: whether the acceptance \& commitment therapy is efficient for decreasing anxiety of the youngster girls?

\section{The research method}

\section{The community, sample and sampling}

The investigation method is of the type semi-test, with a pre-test and post-test plan, on the control group. In this research, the statistical context included all youngster girls at the ages of 14 to 18 , who had referred to a consultative \& psychological service center in the city of Tehran in 2013.

\footnotetext{
${ }^{3}$ General Anxiety Disorder

${ }^{4}$ acceptance \& Commitment Therapy (ACT)

${ }^{5}$ Romer \&Orsillo

${ }^{6}$ Eifert, H. G. ,\& Forsyth
} 
The available sampling method is together with random substitution in the control and test groups. The sample volume for each group in the present study estimated as 15 (subject) people. In this research in order for collecting data, questionnaire was employed.

\section{The scale \& measure for depression and DASS stress}

This scale was developed by Lavy bund and Lavybundin 1995. It has two forms. its main form(this form) includes of 42questions in which every mental structures such as" stress" ، "anxiety", "depression" are evaluated by 14 different question. Sahebi and colleagues (1384) have verified Short questioners form for Iran population that it consists of 21 questions. Studies have been done by Lavy bund and Lavy bund (1995), illustrates that retest reliability for Ancillary infra structures were $81 \%$ for stress $79 \%$ for anxiety, $71 \%$ for depression, respectively. Also, Validity for beek anxiety inventory and beek depression inventory with correlation coefficients, were $81 \%$ and $74 \%$ respectively. Therefore, this scale has suitable reliability to use in research and Diagnostic activities.

\section{Performance method}

Initially, by performing Dass 42 test on female adolescent between 14 and 18 years old who went to health centers, they Randomly divided into two equal groups $(\mathrm{N}=15)$,experimental and control groups, by using of sampling method from 30 female teenagers Those who have symptoms of selection anxiety. In there-test, Doss 42 test on subject's performance, and subjects experimental groupin 12 sessions and 45to 60minutes for each session, exposed to therapy acceptance and commitment, and then in the middle period, above test be checked again as a revision. Finally, the mentioned test was performed as a pre-test in the last session.

\section{Data analysis method}

In this research, based on variables those to be survey ed and the types of collected data and to describe them in central tendency, distribution and Distribution of scores. In the analytical part, due to the nature of measurement scale of interval one and due to three dependent variable measurements before and after therapy for control and experimental groups, split- plot analysis of variance method was used.

But in inferential and analytical step, first difference between anxiety scores in before therapy in the experimental and control groupie order to select the suitable test (analysis of variance for control the effect of pre-test) for two independent group, was tested by using of t- test. 


\section{Hypothesis: reduction of student's anxiety is affected by acceptance and commitment therapy (ACT).}

The following table shows the results of this test. As regards in that level of Meaningful test is higher than 0.05 , thus. It's supposed to be zero where there isn't any different between scores of anxiety in control group and experimental groups this supposition is accepted. Therefore, there is not necessary to Kuryanc analysis in order to control the effect of pretest as covariate variable.

Independent T-test result for test of anxiety scores difference before of control and experimental group therapy

\begin{tabular}{|c|c|c|c|c|c|c|c|}
\hline \multirow{2}{*}{$\begin{array}{l}\text { High } \\
\text { range }\end{array}$} & \multirow{2}{*}{$\begin{array}{l}\text { Low } \\
\text { range }\end{array}$} & \multirow{2}{*}{$\begin{array}{c}\text { Deviation } \\
\text { scale }\end{array}$} & \multirow{2}{*}{$\begin{array}{c}\text { Meaningful } \\
\text { level }\end{array}$} & \multirow{2}{*}{$\begin{array}{c}\text { Free } \\
\text { degree }\end{array}$} & \multirow{2}{*}{$\mathbf{t}$} & \multicolumn{2}{|c|}{ Lyon test } \\
\hline & & & & & & Me & $\begin{array}{l}\text { ingful } \\
\text { el }\end{array}$ \\
\hline 1.003 & -2.603 & 0.880 & -.800 & 0.371 & 28 & -.909 & 0.252 \\
\hline
\end{tabular}

Above Hypothesis result illustrate that level of Meaningful test is higher than 0.05 , thus. It's supposed to be zero where there isn't any different between scores of anxiety in control group and an experimental group, this supposition is accepted.

\section{DISCUSSION AND CONCLUSION}

Results of the research show different between experiment and control groups. According the obtained data, there is a significant difference between both groups in anxiety scores $\left(\mathrm{F}_{1,28}=\right.$ 64601; $\mathrm{p}=0.000)$. In other words, acceptance and commitment therapy has caused reducing stress scores in the experiment group. Eta coefficient (0.698) shows that there are identified about $70 \%$ variance of the dependent variable (anxiety scores) have been explained through interference therapy. Results of the conducted studies by I not and Farsi's (2006) show that state worrying will reduce anxiety in short-term, and it increases anxiety in long-term. Acceptance and commitment therapy with awareness-based exercises (self as field and relationship with now) reduce experimental avoidance and increase acceptance. In the other hand, mixing and cognitive inequality will be increased that it will increase flexibility.

In the other hand, anxiety decreases focusing on valuable objectives. In acceptance and commitment therapy, anxiety will be decreased by increasing experimental avoidance, acceptance and flexibility. It provides proper requirements to focus on valuable objectives. 
Emphasizing, reconstructing and performing values will increase focusing on valuable objectives.

The finding is consistent with the carried out research by Motto (2012). In research of treating main anxiety using acceptance and commitment therapy method, he concluded that it is not a good method to treat people with main anxiety, but increases their mental-social performance. It is also consistent with the carried out researches by Touhig and Hees (2008) and Long Moore and Worl (2007). They studied effectiveness of treating acceptance and commitment therapy on the generalized anxiety disorder. They concluded that acceptance and commitment therapy is effectiveness to treat the disorder.

\section{REFERENCES:}

Kaplan and Sadock's Synopsis of Psychiatry In (1387). Summary of psychiatry. translation by Farzin Rezaei . Tehran, Arjmand.

Barlow DH. Anxiety and Its Disorders: The Nature and Treatment of Anxiety and Panic, 2th ed. New York: Guilford Press; 2002 .p. 65 -75.-American Psychiatric Association Diagnostic and Statistical Manual of Mental Disorders, 4th ed. Text Revision (DSM -IV -TR) Washington DC: American Psychiatric Press; 2000.

-Eifert, H. G. ,\& Forsyth, P. J .(2005). Acceptance \& CommitmentTherapy for anxiety disorders. Oakland: harbinger.

-Long m.re

-Moto (2012)

-Roemer, L. , \&Orsillo, S. M. (2007).An open trial of an acceptancebasedbehavior therapy for generalized anxiety disorder. BehaviorTherapy, 38, 72.85

-Saavadara (2007)

-Twohig \& Hayes 